\title{
A METHOD FOR CHARACTERISING HUMAN INTERVERTEBRAL DISC GLYCOSAMINOGLYCAN DISACCHARIDES USING LIQUID CHROMATOGRAPHY-MASS SPECTROMETRY WITH MULTIPLE REACTION MONITORING
}

\author{
X. Liu ${ }^{1,3}$, D. Krishnamoorthy ${ }^{2}$, L. Lin ${ }^{1}$, P. Xue ${ }^{1}$, F. Zhang ${ }^{1}$, L. Chi ${ }^{3}$, R.J. Linhardt ${ }^{1}$ and J.C. Iatridis ${ }^{2 *}$ \\ ${ }^{1}$ Departments of Chemistry and Chemical Biology, Biological Sciences, Biomedical Engineering and \\ Chemical and Biological Engineering Center for Biotechnology and Interdisciplinary Studies, \\ Rensselaer Polytechnic Institute, Troy, NY, USA. \\ ${ }^{2}$ Leni and Peter W. May Department of Orthopedics, Icahn School of Medicine at Mount Sinai, New York, \\ NY, USA. \\ ${ }^{3}$ National Glycoengineering Research Center, Shandong University, Jinan, Shandong, China
}

${ }^{\S}$ These authors contributed equally to the work.

\begin{abstract}
Intervertebral disc (IVD) degeneration results in the depletion of proteoglycans and glycosaminoglycans (GAGs), which can lead to structural and mechanical loss of IVD function, ingrowth of nociceptive nerve fibres and eventually discogenic pain. Specific GAG types as well as their disaccharide patterns can be predictive of disease and degeneration in several tissues but have not been comprehensively studied within the IVD. A highly sensitive mass spectrometry based technique with multiple reaction monitoring (MRM) was used to provide characterisation of chondroitin sulphate (CS), hyaluronic acid (HA), heparan sulphate (HS) and their disaccharide sulphation patterns across different anatomical regions of human IVDs. Principal component analysis further distinguished important regional variations and proposed potential ageing variations in GAG profiles. CS was the GAG in greatest abundance in the IVD followed by HA and HS. Principal component analysis identified clear separation of GAG profiles between nucleus pulposus and annulus fibrosus in young and old specimens. Distinct patterns of predominantly expressed disaccharides of CS and HS between young and old IVD samples, provided preliminary evidence that important alterations in disaccharides occur within IVDs during ageing. This technique offered a novel approach to identify and quantify specific GAG disaccharides in human IVDs and the data presented were the first to offer insight into the spatial distribution as well as association with ageing of GAGs and GAG disaccharide sulphation patterns across the human IVD.
\end{abstract}

Keywords: Intervertebral disc, disc degeneration, ageing, glycosaminoglycan, chondroitin sulphate, hyaluronic acid, heparan sulphate, mass spectrometry.

*Address for correspondence: James C. Iatridis, Leni \& Peter W. May Department of Orthopaedics, Icahn School of Medicine at Mount Sinai, 1 Gustave Levy Place, Box 1188, New York, NY 10029-6574, USA. Email: james.iatridis@mssm.edu

Copyright policy: This article is distributed in accordance with Creative Commons Attribution Licence (http://creativecommons.org/licenses/by-sa/4.0/).

Abbreviations used

Intervertebral disc (IVD), glycosaminoglycan (GAG), chondroitin sulphate (CS), hyaluronic acid (HA), heparan sulphate (HS), annulus fibrosus (AF), outer anterior annulus fibrosus (AO), inner anterior annulus (AI), nucleus pulposus (NP), inner posterior annulus (PI), outer posterior annulus (PO), inner posterior-lateral annulus (LI), and outer posteriorlateral annulus (LO), principal component analysis (PCA), principal component (PC), multiple reaction monitoring (MRM), liquid chromatography-mass spectrometry (LC-MS)

\section{Introduction}

Low back and neck pain are the leading causes of global disability and among the top causes for absence from work (GBD 2015 Disease and Injury Incidence and Prevalence Collaborators, 2016; Hoy et al., 2010). The US economic costs for back and neck pain are approximately $\$ 253$ billion for direct treatment and lost wages (Dieleman et al., 2016; Gunnar Anderson, 2014; Institute of Medicine (US) Committee on Advancing Pain Research, Care, and Education, 2011). Back and neck pain are strongly associated with intervertebral disc 
(IVD) degeneration. IVD degeneration correlates with the normal process of ageing but also involves structural failure that can arise due to external factors including genetics, environment, metabolic/hormonal changes and lifestyle (Adams and Roughley, 2006; Iatridis et al., 2009; Vo et al., 2016). The causes of pain and disability from IVD degeneration are complex and multifactorial involving biomechanical failure, extracellular matrix degradation, chronic inflammation and neurovascular growth into the normally avascular and aneural IVD (Freemont et al., 1997; Iatridis et al., 2013; Ito and Creemers, 2013; Johnson et al., 2015; Kadow et al., 2015; Mok et al., 2016; Molinos et al., 2015; Samartzis et al., 2013).

IVD degeneration is nearly always characterised by degradation and depletion of the proteoglycan component of the extracellular matrix. Proteoglycans consist of proteins bearing covalently bound polysaccharides that belong to a family of sulphated glycosaminoglycans (GAG) including chondroitin sulphate (CS), keratan sulphate, dermatan sulphate, and heparan sulphate (HS). Hyaluronic acid (HA) is another GAG but is not synthesised on a protein core. Aggrecan is the predominant proteoglycan within the IVD, making up about $65 \%$ of the nucleus pulposus (NP) and 15-20\% of the annulus fibrosus (AF) by dry weight (Gruber et al., 2011; Roughley, 2004). Aggrecan is also the most glycated proteoglycan, consisting mostly of CS and keratan sulphate chains, which form aggregates by interacting with HA (Sivan et al., 2014).

Proteoglycans serve many mechanical functions. The fixed charges of GAGs maintain IVD osmotic pressure and hydration to allow the IVD to withstand high compressive loads (Urban and McMullin, 1988). The loss of proteoglycans can directly result in decreased swelling and reduced water content in the IVD; and this loss of swelling pressure can lead to structural disorganisation, delamination, and annular fissures (Adams et al., 2000; Stokes and Iatridis, 2004). As a result, the total GAG content distribution in the IVD has been characterised with regional position, ageing and degeneration (Antoniou et al., 1996; Iatridis et al., 2007; Melrose et al., 2001; Roughley, 2006). Multiphasic finite element model predictions determine that GAG content and distribution are both important in defining IVD swelling pressure, hydration, mechanics and transport (Barthelemy et al., 2016; Gao et al., 2016; Iatridis et al., 2003; Jackson et al., 2011; Yao and Gu, 2006).

Proteoglycans also serve several biological functions through their interactions with cytokines, cell surface receptors, growth factors as well as other extracellular matrix proteins (Collin et al., 2016; Hayes et al., 2011). The ingrowth of nociceptive nerve fibres and blood vessels has been reported in painful, degenerated IVDs and is suggested to be a source of discogenic pain (Freemont et al., 2002). Interestingly, aggrecan is able to inhibit both endothelial cell adhesion/migration as well as the ingrowth of nerve fibres, and this effect is diminished following enzymatic degradation (Cornejo et al., 2015; Johnson et al., 2002; Johnson et al., 2005; Purmessur et al., 2015). Intact CS is identified as an essential contributor to the anti-angiogenic and anti-neuronal effects of notochordal cell conditioned medium making it an attractive target for inhibiting neurovascular ingrowth in degenerating IVDs (Cornejo et al., 2015; Purmessur et al., 2015).

The sulphation patterns of GAGs can also play important physical and biological roles (Rogers et al., 2011), yet these are surprisingly under-explored in musculoskeletal tissues. Specific patterns of human urinary CS and HS disaccharide sulphations are more predictive of the development/progression of renal dysfunction and hospital mortality than others (Schmidt et al., 2016). Similarly, age- and sex- related variations in CS sulphation in synovial fluid are known to exist (Nakayama et al., 2002). CS disaccharide sulphation also changes during maturation in ovine IVD (Collin et al., 2016). Cumulatively, these studies suggest that GAG disaccharides may change in IVD disease and degeneration. However, the GAG sulphation patterns in human IVDs and their functional changes in ageing and degeneration remain largely unknown motivating the development of new tools for their characterisation.

Most IVD GAG measurements use the dimethylmethylene blue (DMMB) assay and are limited to measurements of total GAG content. Only a few papers describe changes in GAG types and disaccharides in IVDs which use high-performance liquid chromatography (HPLC) or immuno-labelling of GAG epitopes (Collin et al., 2016; Hayes et al., 2011; Melrose et al., 2001; Nakayama et al., 2002; Sivan et al., 2014). It is notable that there is essentially no literature on GAG disaccharides in human IVDs, and methods previously used for their characterisation in IVDs of other species lack the sensitivity of liquid chromatography-mass spectrometry (LC-MS). The current study applied a, highly-sensitive method that combined the physical separation abilities of LC with the mass analysis abilities of MS, along with nontraditional statistical methods to isolate, identify and characterise GAG types and disaccharides in human IVDs with anatomical region. This project focused on methodological validation and characterisation of regional differences. We hypothesised that this novel GAG analytical technique would enable precise measurement of GAG disaccharides that would allow identification of even subtle differences in human IVDs across anatomical region and between specimens. Human IVDs ranged in age from 9-73 years old, with the majority of IVDs being a mature population with moderate degeneration and one very young and healthy sample (9 year old) for comparison (Table 1). Principal component analysis (PCA) was applied as a tool that utilised all the variances obtained from the GAG analyses to identify features that could find correlations in samples that were otherwise indistinguishable by traditional statistical analyses. 
Table 1. Specimen, age, sex, disc degeneration grade and cause of death.

\begin{tabular}{|c|c|c|c|c|}
\hline Specimen & Age & Sex & Thompson Grade & Cause of Death \\
\hline Hu29 & 9 & M & 1 & Cardiac arrest \\
\hline Hu1 & 47 & F & 2 & Respiratory failure \\
\hline Hu4 & 53 & M & 3 & Cardiac arrest \\
\hline Hu18 & 62 & F & 3 & Heart disease \\
\hline Hu0 & 66 & F & 4 & Systolic heart failure \\
\hline Hu30 & 73 & M & $3 / 4$ & Cancer \\
\hline
\end{tabular}

\section{Materials and methods}

\section{Human tissue collection and preparation}

Six totally intact human IVD samples were harvested from cadavers ranging in age from 9-73 years. Lumbar spines were obtained by autopsy services at Mount Sinai Hospital, with permission, and following procedures approved by the Institutional Review Board of Mount Sinai. The age, sex and cause of death of each donor were recorded (Table 1). IVDs were isolated from spinal specimens using a scalpel, and graded morphologically using the Thompson classification, which was modified for transverse sections (Acaroglu et al., 1995; Thompson et al., 1990). After cleaning and removing vertebrae, cartilage endplates and surrounding soft-tissues, each IVD was cut by scalpel into 7 anatomical regions: the nucleus pulposus (NP), posterior inner and outer annulus fibrosus (PI, PO), anterior inner and outer annulus fibrosus (AI, AO) and posterior-lateral inner and outer annulus fibrosus (LI, LO) (Fig. 1). A total of 156 specimens were analysed. 140 samples were obtained from 5 mature IVDs which had each of 7 anatomic regions divided into four equal sections for quadruple measurements. 16 samples were obtained from Hu29 (9 year old) IVD, which was smaller and consequently all $6 \mathrm{AF}$ regions (AO, AI, PI, PI, LI, LO) were divided into two sections for duplicate measurements (12 samples) with sufficient NP tissue for quadruple measurements (4 samples). Wet weights were recorded.

The collected IVD samples were defatted with $0.5 \mathrm{~mL}$ acetone (Sigma-Aldrich, St. Louis, MO, USA) vortex for $30 \mathrm{~min}$, and dried in the hood before digestion. The defatted samples were suspended in $0.85 \mathrm{~mL}$ of water with $0.15 \mathrm{~mL}$ of actinase E $(5 \mathrm{mg} / \mathrm{mL}$,

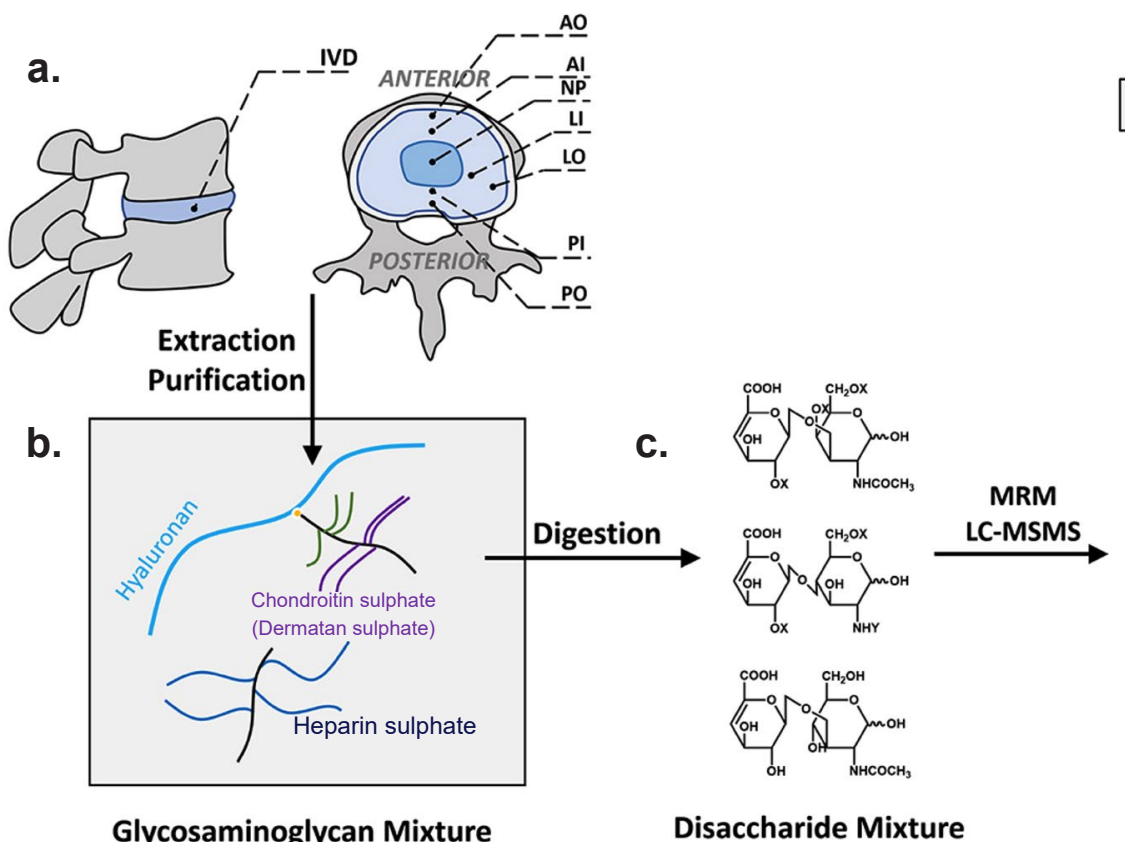

Differences and Relationships

Fig. 1. Flow chart for human intervertebral disc (IVD) specimen treatment for glycosaminoglycan disaccharide composition analyses. (a) 7 regions tested in each IVD: outer anterior annulus (AO), inner anterior annulus $(\mathrm{AI})$, nucleus pulposus (NP), inner posterior annulus (PI), outer posterior annulus (PO), inner posteriorlateral annulus (LI), and outer posterior-lateral annulus (LO). (b) Glycosaminoglycans purified from the IVD regions, digested by the heparinases and chondroitinase ABC. (c) Structures of the disaccharides after adding heparinase I/II/III and chondroitinase ABC. $\triangle \mathrm{UA2X}(1,3)$ GalNAc4X6X is generated from chondroitin sulphate (CS). $\triangle \mathrm{UA2X}(1,4)$ GlcNY6X is generated from heparan sulphate (HS). $\triangle \mathrm{UA}(1,3)$ GlcNAc is generated from hyaluronic acid (HA). $\mathrm{X}=\mathrm{H}$ or $\mathrm{SO}_{3} \mathrm{H}$. $\mathrm{Y}=\mathrm{H}$ or $\mathrm{SO}_{3} \mathrm{H}$ or $\mathrm{COCH}_{3}$. (d) Extracted ion chromatograms (EICs) of the disaccharides. 
Kaken Biochemicals, Tokyo, Japan) for proteolysis at $55{ }^{\circ} \mathrm{C}$, until all the tissues were dissolved $(36 \mathrm{~h})$. Refer to Zhao et al. (2012) for validation and percentage recovery from the following GAG purification and desalting methods. GAGs were purified using Vivapure Q Mini H spin columns (Sartorious Stedim Biotec, Bohemia, NY, USA). Component eluted was then desalted by passing through a $3 \mathrm{kDa}$ molecular weight cut off (MWCO) spin column (Millipore, Bedford, MA, USA) and washed twice with distilled water. The casing tubes were replaced, before $150 \mu \mathrm{L}$ of digestion buffer ( $50 \mathrm{mM}$ ammonium acetate containing $2 \mathrm{mM}$ calcium chloride adjusted to $\mathrm{pH}$ 7.0, Fisher Scientific, Springfield, NJ, USA) was added to the filter unit. Recombinant heparin lyase I, II, III (pH 7.0-7.5) and recombinant chondroitin lyase ABC (10 mU each, pH 7.4) were added to each sample and mixed well (Su et al., 1996). The samples were all placed in a water bath at $37^{\circ} \mathrm{C}$ for $12 \mathrm{~h}$, after which enzymatic digestion was terminated by removing the enzymes through centrifugation. The filter unit was washed twice with $100 \mu \mathrm{L}$ distilled water and the filtrates containing the disaccharide products were lyophilised.

The dried disaccharide samples were 2-aminoacridone (AMAC)-labelled (AMAC from Sigma-Aldrich, St. Louis, MO) by adding $10 \mu \mathrm{L}$ of $0.1 \mathrm{M}$ AMAC in dimethyl sulphoxide/acetic acid $(17 / 3, V / V)$ incubating at room temperature for $10 \mathrm{~min}$, followed by adding $10 \mu \mathrm{L}$ of $1 \mathrm{M}$ aqueous sodium cyanoborohydride (Sigma-Aldrich (St. Louis, MO) and incubating for $1 \mathrm{~h}$ at $45^{\circ} \mathrm{C}$. A mixture containing all 17 CS, HS and HA disaccharide standards (Iduron, Manchester, U.K., see Table 2 for structures) prepared at $6.25 \mathrm{ng} / \mu \mathrm{L}$ was similarly AMAC-labelled and used for each run as an external standard. After the AMAClabelling reaction, the samples were centrifuged and each supernatant was recovered.

\section{LC-MS/MS analysis}

The disaccharide analysis was performed as previously described (Sun et al., 2015). LC was performed on an Agilent $1200 \mathrm{LC}$ system at $45^{\circ} \mathrm{C}$ using an Agilent Poroshell 120 ECC18 (2.7 $\mu \mathrm{m}$, $3.0 \times 50 \mathrm{~mm}$ ) column. A triple quadruple mass spectrometry system equipped with an ESI source (Thermo Fisher Scientific, San Jose, CA) was used as the detector. The online MS analysis was done in the multiple reaction-monitoring (MRM) mode.

\section{Principal component analysis}

PCA is an analytical method that uses multivariate data to differentiate between observations by transforming the data to a new coordinate system. The new coordinates, which are in $n$-dimension, are the principal components (PCs). The first coordinate, which provides the greatest variance, is called the first PC. The further PCs provide less variance than their preceding PCs. PCA can therefore be helpful in analysing high dimensional datasets (i.e. datasets with many sources of variation and output measurements) with dimensionality reduction that allow visualisation of PCs that highlight the greatest variance. Such methods were previously used to characterise complex datasets with large numbers of experimental variables characterising heparin (Liu et al., 2017; Zhao et al., 2015). Two sets of PCA were operated on software Origin 9.1 (OriginLab). A 2D PCA was performed to visualise all IVD regions and replicates to obtain an overview plot and to determine if GAG values were best distinguished by human subject (which vary by age), circumferential regional position or radial regional position. Percentages from total GAG composition, HS disaccharide composition and CS composition were chosen as variables. Two PCs were extracted where all the tested samples (156 total samples from 7 IVD regions) were observed. A 3D PCA was then performed to determine, with better resolution, if GAG values were distinct by IVD regional position. In this second set of PCA, 15 variables were the HS, CS, and HA concentrations from total GAG composition of five patients (Hu29 was excluded because of its smaller number of replicates). Three PCs were extracted from the 15 variables. Seven different IVD regions, analysed in quadruple (biological replicates), were chosen as the observations.

Table 2. Chondroitin sulphate, heparan sulphate and hyaluronic acid disaccharide structures analysed by LC-MS

\begin{tabular}{|c|c|}
\hline \multicolumn{2}{|c|}{ CS disaccharides } \\
\hline TriS $_{\mathrm{CS}}$ & $\Delta \mathrm{UA} 2 \mathrm{~S}(1,3)$ GalNAc4S6S \\
\hline $2 \mathrm{~S}_{4} \mathrm{~S}_{\mathrm{CS}}$ & $\triangle \mathrm{UA} 2 \mathrm{~S}(1,3)$ GalNAc4S \\
\hline $2 \mathrm{~S}^{2} \mathrm{~S}_{\mathrm{CS}}$ & $\triangle \mathrm{UA2S}(1,3)$ GalNAc6S \\
\hline $4 \mathrm{~S} 6 \mathrm{~S}_{\mathrm{CS}}$ & $\Delta \mathrm{UA}(1,3) \mathrm{GalNAc4S6S}$ \\
\hline $2 S_{C S}$ & $\Delta \mathrm{UA} 2 \mathrm{~S}(1,3)$ GalNAc \\
\hline $4 S_{\mathrm{CS}}$ & $\Delta \mathrm{UA}(1,3) \mathrm{GalNAc} 4 \mathrm{~S}$ \\
\hline $6 \mathrm{~S}_{\mathrm{CS}}$ & $\Delta \mathrm{UA}(1,3)$ GalNAc6S \\
\hline $0_{\mathrm{SC}} \mathrm{S}$ & $\Delta \mathrm{UA}(1,3) \mathrm{GalNAc}$ \\
\hline \multicolumn{2}{|r|}{ HS disaccharides } \\
\hline TriS $_{\mathrm{HS}}$ & $\Delta \mathrm{UA2S}(1,4)$ GlcNS6S \\
\hline $\mathrm{NS6S}_{\mathrm{HS}}$ & $\Delta \mathrm{UA}(1,4) \mathrm{GlcNS6S}$ \\
\hline $\mathrm{NS}_{2} \mathrm{~S}_{\mathrm{HS}}$ & $\Delta \mathrm{UA} 2 \mathrm{~S}(1,4) \mathrm{GlcNS}$ \\
\hline $\mathrm{NS}_{\mathrm{HS}}$ & $\Delta \mathrm{UA}(1,4) \mathrm{GlcNS}$ \\
\hline $2 \mathrm{~S}^{2} \mathrm{~S}_{\mathrm{HS}}$ & $\Delta \mathrm{UA2S}(1,4)$ GlcNAc6S \\
\hline $6 \mathrm{~S}_{\mathrm{HS}}$ & $\Delta \mathrm{UA}(1,4)$ GlcNAc6S \\
\hline $2 \mathrm{~S}_{\mathrm{HS}}$ & $\Delta \mathrm{UA2S}(1,4)$ GlcNAc \\
\hline $0 \mathrm{~S}_{\mathrm{HS}}$ & $\Delta \mathrm{UA}(1,4) \mathrm{GlcNAc}$ \\
\hline \multicolumn{2}{|c|}{ HA disaccharides } \\
\hline $0 \mathrm{~S}_{\mathrm{HA}}$ & $\Delta \mathrm{UA}(1,3) \mathrm{GlcNAc}$ \\
\hline
\end{tabular}




\section{Statistical analysis}

All values were represented as the mean \pm standard deviation. One-Way ANOVA or Student's t-test was used to determine significant differences between regions and GAG type. The mature group consisted of 5 IVDs ranging in age from 47-73 years old. The young age group was represented by one IVD sample, age 9 years old. Average of all 6 samples together was also represented. Data were considered significant if $p<0.05$.

\section{Results}

\section{Distribution of CS, HA and HS GAG types across IVD regions}

Each of the three primary GAG types (CS, HA and HS) was averaged across 6 specimens, within each of the 7 regions analysed. Comparisons between GAG types within each region confirmed that CS was the most abundant GAG component followed by $\mathrm{HA}$ and $\mathrm{HS}$ in all regions analysed, whether calculated as percent composition or total dryweight (Fig. 2). No significant regional differences in GAG composition were identified, although trends indicated approximately $50 \%(p=0.2)$ greater CS per dry weight in the NP and PI regions compared to the outer AF regions.

While the average GAG content of the 6 specimens together did not clearly show region specificity, separating mature specimens from immature ones more clearly revealed the variability in distribution of CS, HA and HS across regions of the IVD. Mature samples (age 40-80 years, $n=5$ ) showed very little variability in CS and HS content, which was evenly distributed across all 7 regions (Fig. 3). HA content was greatest towards the middle IVD regions
$(\mathrm{NP}+\mathrm{PI})$, with least values in the outer AF regions. In contrast, the young specimen, showed a very distinct GAG profile in relation to IVD region with substantially more CS and HS in the NP + PI regions and much smaller amounts in the outer AF (AO, PO, \& LO). HA showed a similar pattern of increasing trend toward inner disc regions, yet HA content was less in all regions for the young than for the mature samples.

GAG values for AF and NP regions were compared to more prominently highlighted regional differences across specimens (Fig. 4). It was notable that the \% CS, HA and HS did not change with anatomical region, highlighting that the \% GAG is largely preserved with age and region. The GAG/dry weight was greatest in the NP and lowest in the outer AF regions, but this trend was not uniform across all specimens and GAG types. Notably, CS and HS had significantly more GAG/dry weight in the NP than AF only in samples with more total GAG content, suggesting that GAG is lost predominantly in the NP for most mature samples. HA GAG/dry weight was surprisingly not greatest in the samples with greatest total GAG content, but instead had largest values in the NP region of a 52 year-old subject, suggesting a pattern that was distinct from ageing.

\section{Regional and age-related changes in CS and HS disaccharides}

Eight CS and HS disaccharides were quantified in the NP and AF of each disc. The data revealed two predominantly expressed disaccharides of each GAG type in both NP and AF: C4S, C6S, H0S and H2S. The distribution of these disaccharides within the NP and AF revealed region and potential age-related associations. While the concentrations of C4S and C6S were similar in NP (Fig. 5a), there was an almost $50 \%$ a. $\% \mathrm{CS}, \mathrm{HA}$ and HS

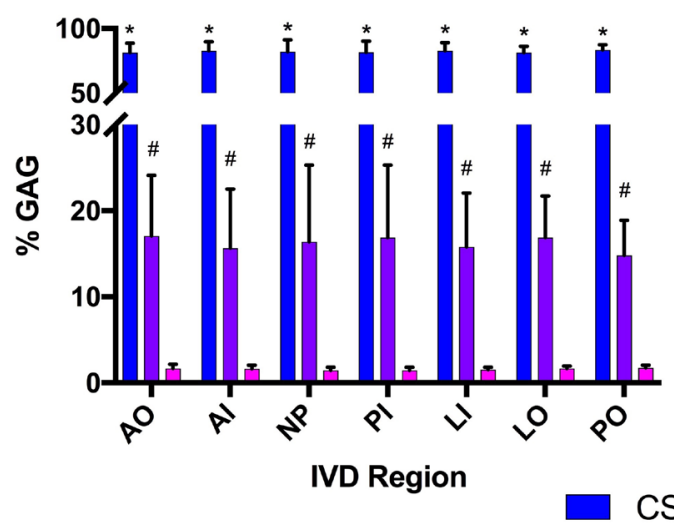

b.

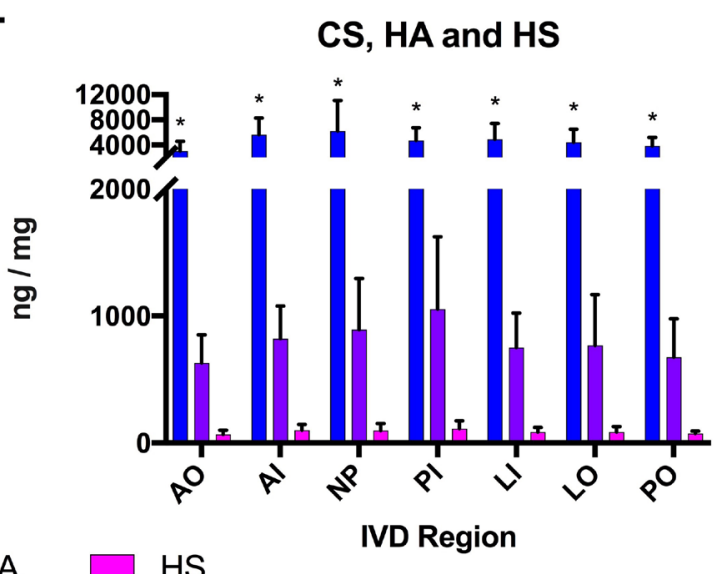

Fig. 2. Composition of GAGs in different regions of the IVDs. (a) Percentage of each GAG type out of total GAG content. Percent of Chondroitin sulphate (CS), Hyaluronic acid (HA), and Heparan sulphate (HS) in the outer anterior annulus (AO), inner anterior annulus (AI), nucleus pulposus (NP), inner posterior annulus (PI), outer posterior annulus (PO), inner posterior-lateral annulus (LI), and outer posterior-lateral annulus (LO). (b) Total GAG composition quantified as ng GAG/mg of dry tissue weight. Data are averaged across all 6 IVDs $(n=6)$ for each anatomical region of interest. One-Way ANOVA between GAG types within each region shows significance "compared to HA and HS if $p<0.05$ and "compared to HS if $p<0.05$. Mean \pm SD. 

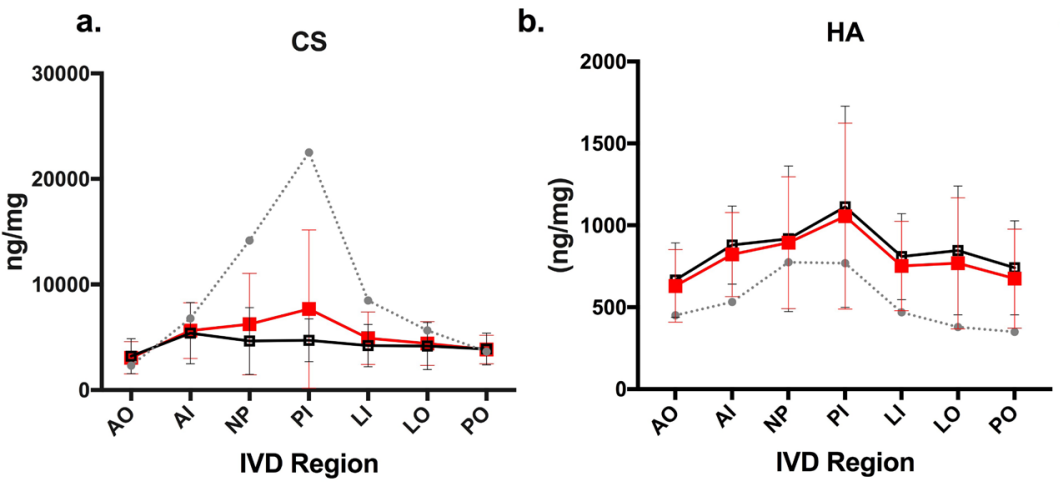

C.

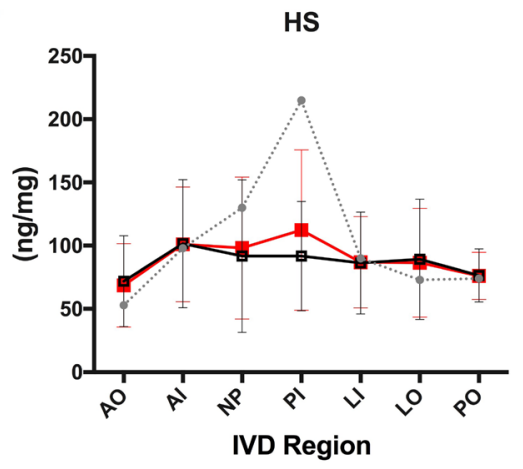

.... young, 9 year $(n=1) \nleftarrow$ mature, 40-80 year $(n=5) \rightarrow$ mean $(n=6)$

Fig. 3. Concentrations of CS, HA and HS, in ng GAG/mg of dry tissue weight, across the 7 regions of the disc. The grey dotted line represents the GAG composition of the young (9 year old) specimen $(n=1)$, mean of biological replicates for each region; the red line represents the average of all IVD specimens, mean $\pm \mathrm{SD}$ $(n=6)$; the black solid line represents the average of all specimens except the one young (9 year old) IVD, mean \pm SD ( $n=5$ mature specimens) for each region.

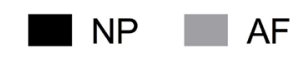

a.

$\%$ CS

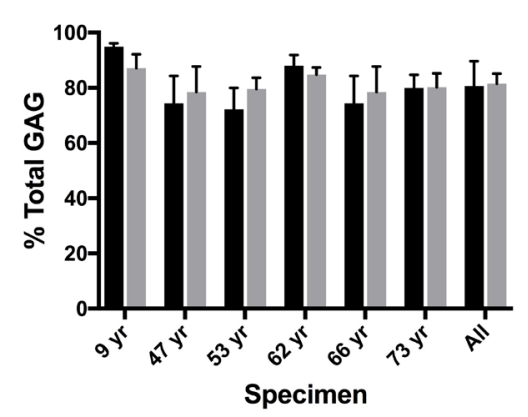

d.

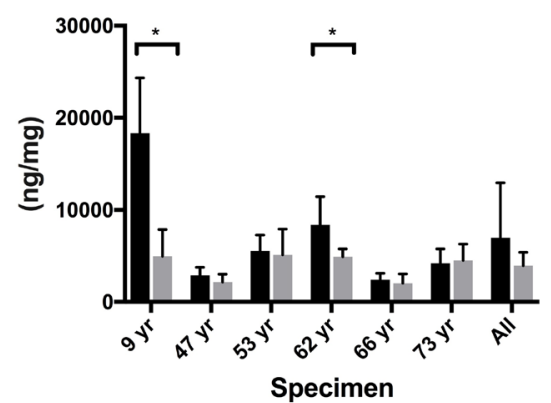

b.

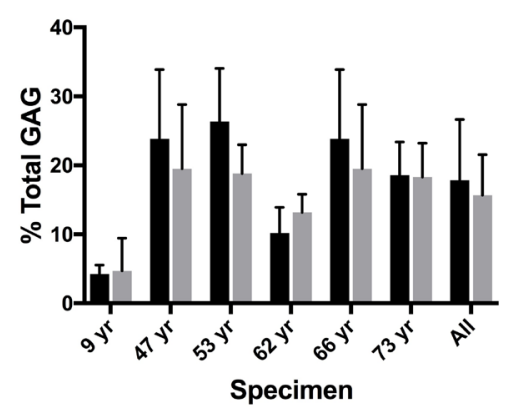

e.

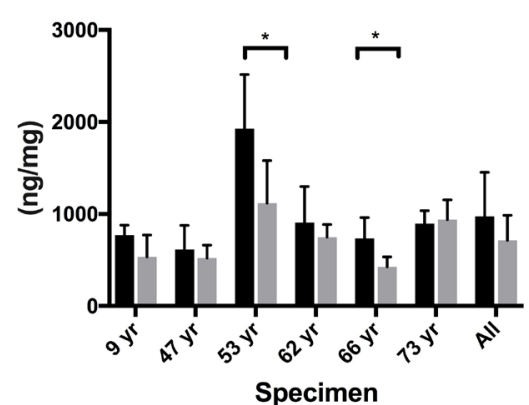

c.

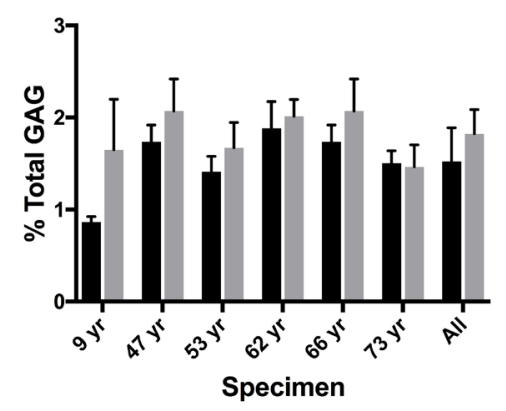

f.

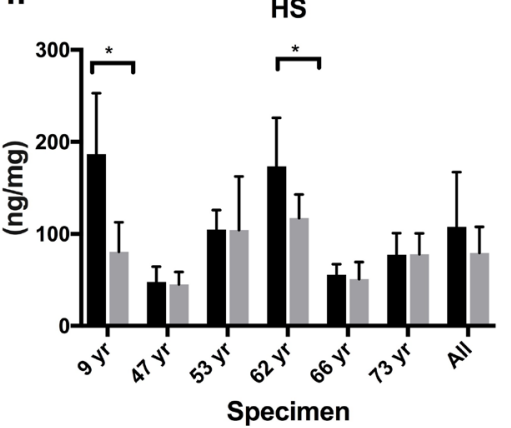

Fig. 4. (a-c) Percentage of CS, HA and HS out of total GAG in the NP and outer AF regions (AO, PO and LO) in each of 6 IVD specimens. (d-f) Total CS, HA and HS represented as ng GAG/mg dry tissue weight in $\mathrm{NP}$ and outer AF regions (AO,PO,LO) in each IVD. For the 9 year old IVD, NP measurements are averaged across $n=4$ biological replicates and AF measurements are averaged across $n=2$ biological replicates. For all other IVDs NP and AF measurements are averaged across $n=4$ biological replicates. Students $t$-test between $\mathrm{NP}$ and AF indicate significance if ${ }^{*} p<0.05$. The average $\%$ GAG and GAG by dry weight of all 6 specimens is represented in the last two bars of each graph, labelled as "All".

Table 3. C6S:C4S ratio within the NP and AF of the young, 9 year old IVD, mean of the mature, 40-80 year old IVD, and mean of all 6 IVD specimens. The C6S:C4S ratio is greater in the mature specimens compared to the young IVD in both the NP and AF.

\begin{tabular}{|l|c|c|c|}
\hline & young, 9 year $(\boldsymbol{n}=\mathbf{1})$ & mature, $\mathbf{4 0 - 8 0}$ year $(\boldsymbol{n}=\mathbf{5})$ & mean $(\boldsymbol{n}=\mathbf{6})$ \\
\hline NP & 0.44 & 1.82 & 1.59 \\
\hline AF & 0.48 & 2.22 & 1.99 \\
\hline
\end{tabular}



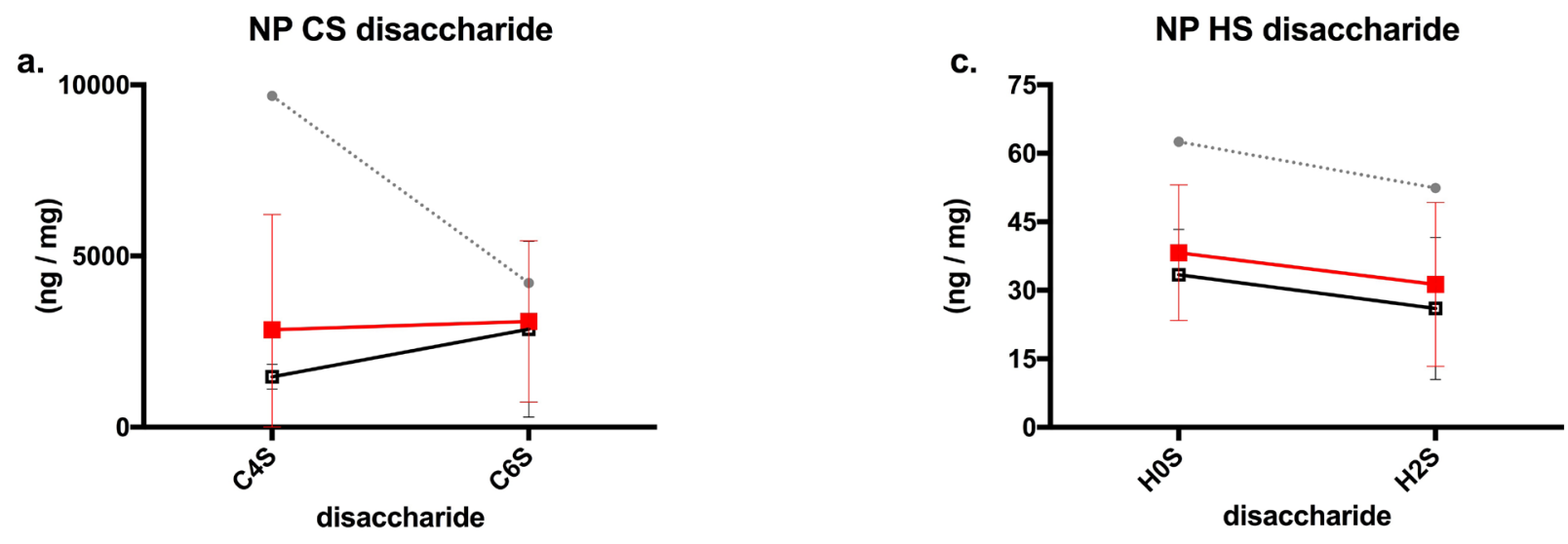

.*..young, 9 year $(n=1) \rightarrow$ mature, $40-80$ year $(n=5)-$ mean $(n=6)$

b.

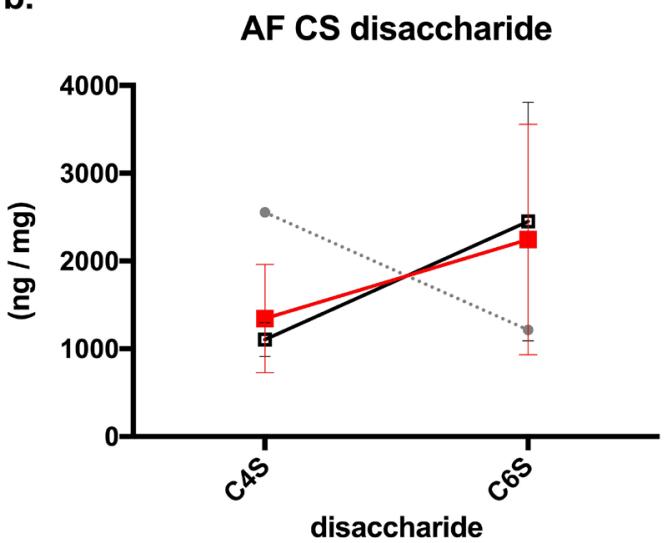

d.

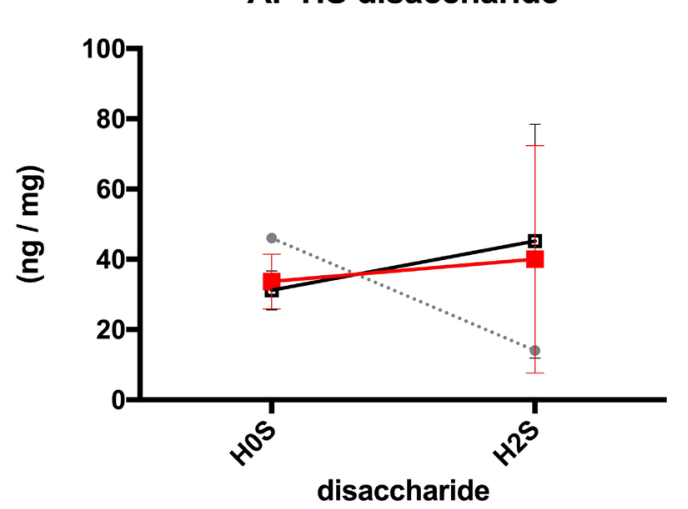

Fig. 5. CS disaccharides, C4S and C6S, in the (a) NP and (b) AF. HS disaccharides, H0S and H2S, in the (c) NP (d) AF. Grey dotted line represents the GAG composition of the young specimen, $n=1$. Red line represents the average of all IVDs, $n=6$. The black solid line represents the average of all IVDs except the young 9 year old IVD, $n=5$. Data are represented as mean \pm SD for the GAG content in ng GAG/mg dry tissue weight for the red and black lines. The grey dotted line represents the average of the biological replicates for the one 9 year old IVD in the NP and AF.

greater amount of C6S compared to C4S in the AF (Fig. 5b), suggesting a change in sulphation position favouring C6S in human IVD AF. The C6S:C4S ratio also supported this trend (Table 3).

Separating the young specimen from the mature specimens exhibited potential age-related associations in CS sulphation patterns. In particular, the young specimen exhibited the greatest C4S in the $\mathrm{NP}$ and AF regions while the mature samples had $76 \%(p=0.06)$ more C6S than C4S in the AF (Fig. 5). It is also notable that while there was no difference in C4S content in the mature NP and AF regions, there was also the least amount of variance compared to that of C6S.

HS disaccharide content showed similar, but more subtle, differences within the NP and AF and between the young and mature specimens when examining closely the two predominant HS disaccharides, HOS and H2S. The mean HS disaccharide content of all six IVDs and only the five mature IVDs exhibited no notable differences in H0S and H2S in either the NP or AF regions. However, there was substantially more $\mathrm{H} 0 \mathrm{~S}$ and H2S in the NP of the young sample than in mature IVDs.

\section{Principal component analysis}

GAG composition and disaccharides showed limited statistical variations with age or region, using traditional statistical analyses (Fig. 2-5). Principal component analysis (PCA) was then applied, as a tool that utilises all the variances obtained from the MRM LC-MS analyses, to identify structural features that could be used to find correlations between the IVD samples based on human subject and anatomical regions that were difficult to identify using traditional statistical analyses. Two PCs were chosen for the first set of PCA, which input all the disaccharide compositional information as either the input variables (total GAG, HS disaccharide and CS disaccharide compositions) or included all the IVD samples as individual observations, without any assignments. The loading plot, Fig. 6a, revealed which 

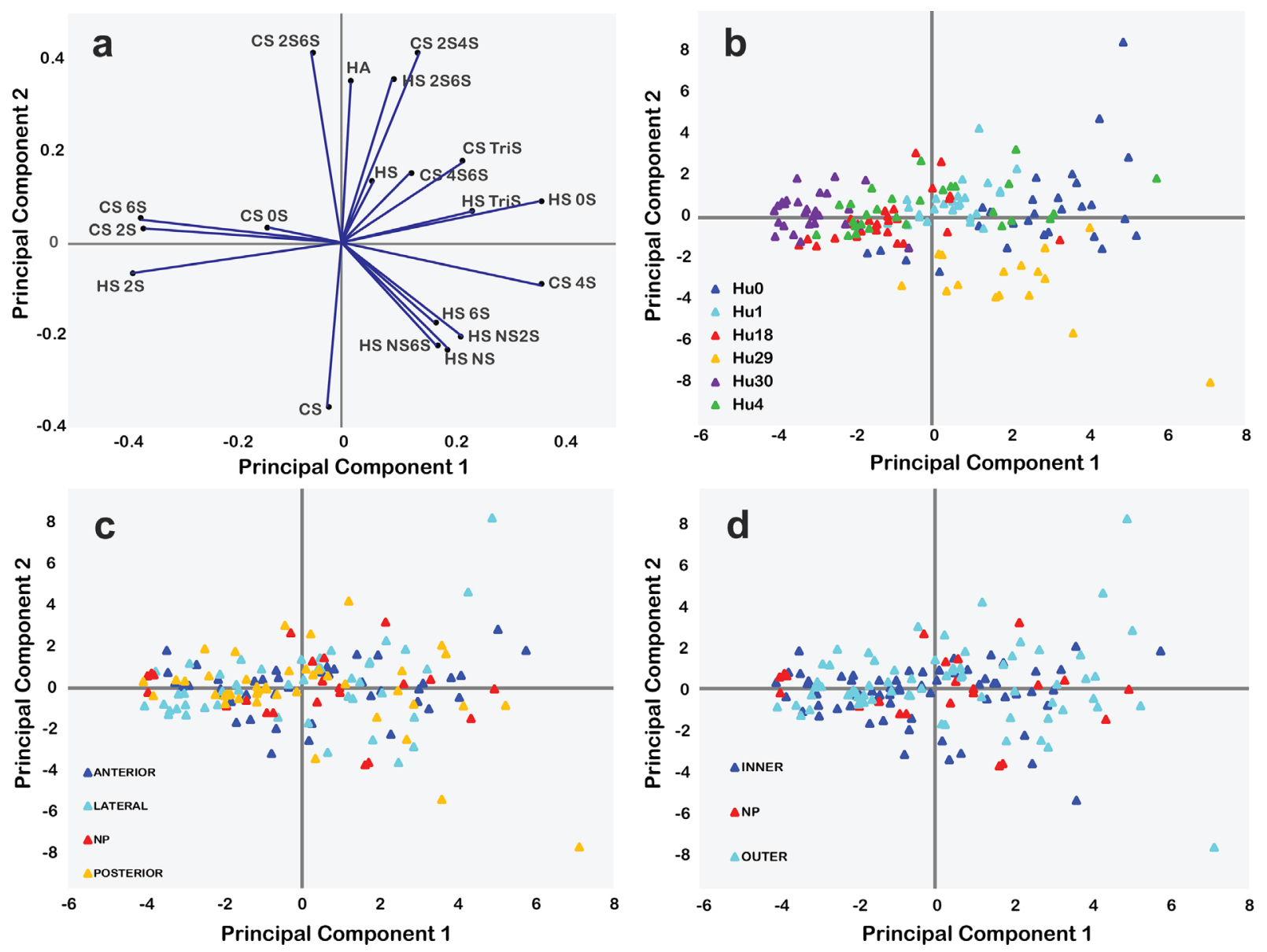

Fig. 6. Principal component analysis (PCA) of all the IVD samples from different patients based on their total GAG composition, HS disaccharide composition and CS disaccharide composition. (a) PCA loadings indicating the coefficients of principal component (PC) 1 and PC2 for the GAG components and the disaccharide components. (b) PCA scores of the IVD samples on principal component (PC) 1 and PC2. Samples were grouped by patients. (c) PCA scores of the IVD samples on PC 1 and PC2. Samples were grouped for different regions by colour considering anterior / lateral / posterior AF regions or nucleus pulposus (NP) regions. (d) PCA scores of the IVD samples on PC 1 and PC2, samples were grouped by inner / outer AF regions or the NP regions.

of the input variables were influential. For example, the inner anterior annulus fibrosus sample NO.1 from patient $\mathrm{Hu} 0$, located at [(PC1, -0.663), (PC2, -2.028)], suggested dominant variables with negative PC1 coefficient values, such as (the percentage of CS in total GAG composition, the percentage of $2 S$ in HS disaccharide composition, the percentages of 2S6S, 6S, 2S, 0S in CS disaccharide composition) and dominant variables with negative PC2 coefficient values, such as the percentage of CS in total GAG composition, the percentage of NS6S, NS2S, NS, 6S, $2 S$ in HS disaccharide composition, the percentages of $4 S$ in CS disaccharide composition, were at larger amounts in the GAG composition or disaccharide composition. Among all these variables, ones with both negative PC1 coefficient values and negative PC2 coefficient values, such as the percentage of $2 S$ in HS disaccharide composition and the percentage of CS in the total GAG composition, were at the largest amounts. The score value plots (Fig. $6 \mathbf{b}, \mathbf{c}, \mathbf{d}$ ), gave the amounts of the PCs in each of the different observations. PC1 and PC2 were the variable combinations of the percentage composition of the disaccharide components and the GAG components. As a result, each IVD sample had its PCA scores related to its GAG compositional information. In the initial steps of the PCA, all the IVD samples were analysed without any assignments. After the scores were output as 156 unlabelled points, the points were grouped based on different patients. IVD samples from each patient were labelled a different colour (Fig. $6 b)$. Samples were then grouped for different regions by colour, considering anterior / lateral / posterior AF regions or nucleus pulposus (NP) regions (Fig. 6c), or grouped by inner / outer AF regions or the NP regions (Fig. 6d). IVD samples from identical patients clustered together in the score value plot (Fig. 6b) showing both PC1 and PC2 values were not far from each other. For example, IVD samples from Hu30 all had negative PC1 values and PC2 values around 0, which indicated that samples from Hu30, 6S, 2S, and $0 S$ were at larger amounts in CS disaccharide 
a

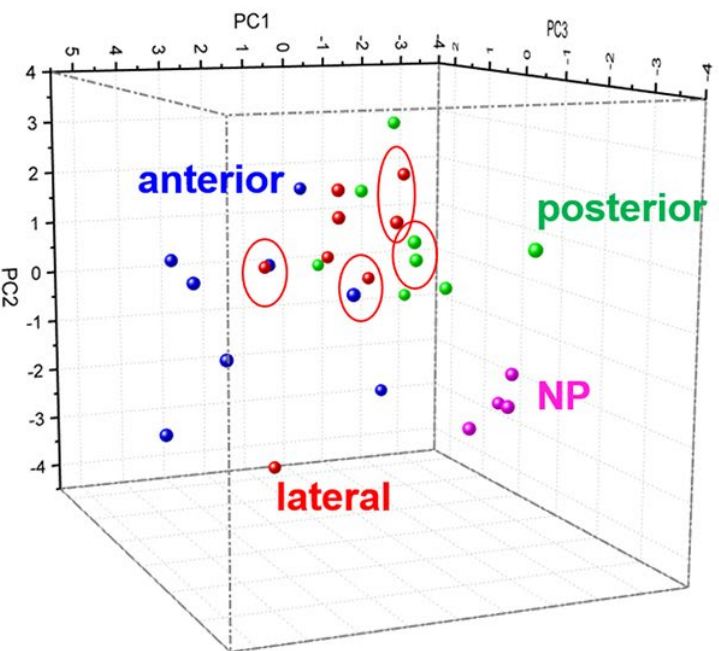

b

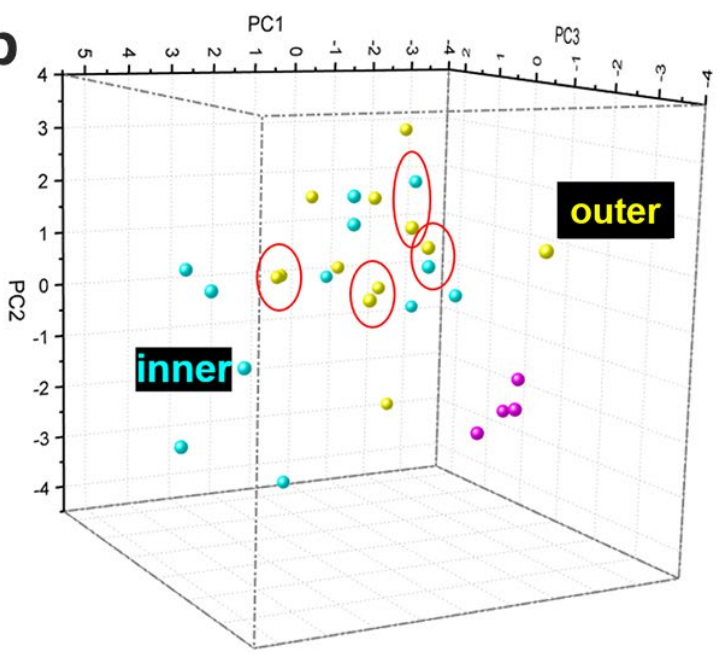

Fig. 7. Principal component analysis (PCA) of the IVD samples from different IVD regions based on the total GAG compositions. Panel (a) and (b) are the same set of results distinguished by different groups. (a) Posterior, anterior and lateral AF regions were highlighted. (b) Inner and outer AF regions were highlighted.

Table 4. Coefficients of three PCs used to identify IVD regions based on GAG composition from each patient.

\begin{tabular}{|c|c|c|c|}
\hline & Coefficients of PC1 & Coefficients of PC2 & Coefficients of PC3 \\
\hline Hu0 CS & 0.268 & 0.193 & -0.399 \\
\hline Hu1 CS & -0.317 & 0.039 & -0.431 \\
\hline Hu4 CS & 0.386 & 0.118 & -0.053 \\
\hline Hu18 CS & -0.178 & -0.392 & -0.256 \\
\hline Hu30 CS & -0.202 & 0.421 & -0.005 \\
\hline Hu0 HA & -0.259 & -0.204 & 0.394 \\
\hline Hu1 HA & 0.323 & -0.057 & 0.425 \\
\hline Hu4 HA & -0.386 & -0.118 & 0.064 \\
\hline Hu18 HA & 0.182 & 0.405 & 0.242 \\
\hline Hu30 HA & 0.186 & -0.421 & 0.004 \\
\hline Hu0 HS & -0.245 & 0.233 & 0.167 \\
\hline Hu1 HS & -0.124 & 0.317 & 0.073 \\
\hline Hu4 HS & 0.165 & 0.044 & -0.353 \\
\hline Hu18 HS & -0.045 & -0.154 & 0.173 \\
\hline Hu30 HS & 0.338 & -0.188 & 0.012 \\
\hline
\end{tabular}

composition and $2 \mathrm{~S}$ was at larger amount in HS disaccharide composition. Similarly, IVD samples from patient $\mathrm{Hu} 29$ had positive PC1 values and negative PC2 values, which indicated that $4 S_{C S}$ and

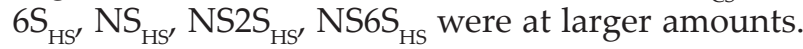
The two most distinguishable subjects, Hu30 and Hu29, were the oldest and youngest among all 6 IVD samples, suggesting important ageing effects on IVD GAG composition, even though the other samples overlapped. IVD regions were not distinguishable using 2 PCs by the anterior / lateral / posterior AF regions or the nucleus pulposus (NP) regions, and the inner / outer AF regions or the NP regions. By comparing the 3 kinds of different groupings (Fig. $6 \mathbf{b}, \mathbf{c}, \mathbf{d})$, it was concluded that IVD samples were more distinguishable on different human beings than on different IVD regions.

Identification of IVD regions, based on GAG composition, was successful by using three PCs on a second set of PCA to increase resolution of regional differences. The GAG composition differences caused by differences between patients were neglected by reconstructing variables from different patients together (Table 4). Samples from anterior, posterior, lateral $\mathrm{AF}$ regions or NP regions were labelled in different colours, (Fig. 7a) samples from inner, outer AF regions or NP regions were also labelled in different colours (Fig. 7b). The coefficient values of the 15 variables (Table 4) and the scores of the 28 observations (Table 5) provided each data point with its PCA scores, related to its GAG compositional information to generate two 3D plots (Fig. 7a,b). Using three PCs IVD samples from NP regions were clearly identifiable from AF regions. Furthermore, the $\mathrm{AF}$ regions were better resolved as compared with the 2D plots using 2 PCs. However, some crosses between different AF regions were observed (red circles). Comparing the groupings in the $3 \mathrm{D}$ plots, we could 
Table 5. PCA scores from 28 observations of the inner and outer anterior/posterior/lateral AF regions and NP region used to generate the 3D plots in Fig. 7.

\begin{tabular}{|l|c|c|c|}
\hline & Scores (PC1) & Scores (PC2) & Scores (PC3) \\
\hline AI 1 & 3.718 & 0.534 & 0.845 \\
\hline AI 2 & 3.885 & 0.381 & -0.267 \\
\hline AI 3 & 3.589 & -0.878 & -0.923 \\
\hline AI 4 & 4.448 & -2.478 & -0.156 \\
\hline LI 1 & 0.653 & 1.449 & -0.266 \\
\hline LI 2 & 0.735 & 1.993 & -0.357 \\
\hline LI 3 & -1.582 & 2.032 & 0.633 \\
\hline LI 4 & 1.314 & -3.799 & 0.905 \\
\hline PI 1 & -2.690 & -0.797 & 1.936 \\
\hline PI 2 & -0.822 & -0.174 & 2.411 \\
\hline PI 3 & -1.754 & 0.000 & -0.402 \\
\hline PI 4 & -0.728 & 0.689 & -0.781 \\
\hline NP 1 & -3.048 & -2.359 & -0.433 \\
\hline NP 2 & -2.378 & -1.389 & -1.491 \\
\hline NP 3 & -1.184 & -2.245 & -1.734 \\
\hline NP 4 & -1.495 & -1.645 & -2.348 \\
\hline AO 1 & 0.145 & 1.622 & 1.691 \\
\hline AO 2 & 1.125 & 0.177 & 1.359 \\
\hline AO 3 & -1.926 & -2.733 & 1.718 \\
\hline AO 4 & 1.200 & 0.318 & -1.444 \\
\hline LO 1 & 0.100 & 1.542 & -1.248 \\
\hline LO 2 & -1.019 & -0.110 & 0.993 \\
\hline LO 3 & -0.425 & 0.218 & 1.540 \\
\hline LO 4 & 1.334 & 0.185 & 1.202 \\
\hline PO 1 & -0.773 & 1.544 & -3.677 \\
\hline PO 2 & -0.337 & 1.146 & -1.204 \\
\hline PO 3 & -0.325 & 1.805 & 0.329 \\
\hline PO 4 & -1.759 & 2.972 & 1.166 \\
\hline & & & \\
\hline
\end{tabular}

conclude that they got similar PC scores because they were either from the same anterior/posterior/lateral regions or the same inner/outer regions. However, both analyses distinguished NP regions from other AF regions.

\section{Discussion}

Proteoglycans play a crucial role in IVDs, by maintaining healthy biomechanical behaviours and performing bioactive functions including the inhibition of neurovascular ingrowth that can cause painful conditions (Adams and Roughley, 2006; Iatridis et al., 2013; Purmessur et al., 2013; Vo et al., 2016). About $90 \%$ of the molecular mass of proteoglycans, including aggrecan, consists of GAGs so that most proteoglycan analyses in the IVD focus on total GAG content. However, GAG types and their disaccharide sequences are also important since they affect interactions with other macromolecules that influence multiple biological processes (Kiani et al., 2002). They can also predict the progression or development of disease and degeneration in various tissues (Collin et al., 2016; Schmidt et al., 2016; Uesaka et al., 2002). The current study characterised, for the first time, GAG types and disaccharides in human IVDs using a sophisticated and sensitive mass spectrometry based technique. IVD specimens ranged from 9-73 years old, and each IVD was analysed in 7 distinct regions that included the NP as well as inner and outer portions of the AF in the anterior, posterior and lateral regions. The focus was to use LC-MS with MRM and PCA to identify variances associated with different anatomical regions in the human IVD. Most IVDs were moderately degenerated from mature humans (47-73 years old), although a single sample with Thompson grade 1 (9 years old) was included to highlight potential differences with degeneration and ageing.

Analysis of GAG types showed CS as the most abundant GAG, followed by HA and HS in all IVD regions. Most of these mature IVD samples had similar amounts of CS, HA and HS in the NP and $\mathrm{AF}$, although differences in the composition within the NP compared to AF regions was most evident in the young specimen as compared to the mature specimens. These data were generally consistent with literature that show greatest GAG content in the NP region and inner AF samples, particularly for young samples (Antoniou et al., 1996; Iatridis et al., 2007). Antoniou et al. also report significantly greater amounts of GAG in the NP than outer AF regions for older IVD specimens (Antoniou et al., 1996), in slight contrast to data reported here. It is likely that the less prominent regional differences found were due to the use of older subjects and the use of larger tissue samples that encompassed a greater radial variation than other data sets (Antoniou et al., 1996; Iatridis et al., 2007). Even with our less prominent regional variations, PCA was demonstrated to be a powerful tool capable of strong distinction of GAG profiles between NP and AF regions. Furthermore, PCA also allowed relatively good distinction of GAG profiles from different human specimens. An interesting regional observation was that multiple GAG types had greater content in PI than NP regions, which could only partly be explained by the inclusion of some NP tissue in the large PI samples. The lower value of GAG content in NP than PI region for multiple measurements in the current study was consistent with heterogeneities previously observed in the mature IVD samples. Specifically, a small 'dip' in total GAG is observed in the central NP region of multiple mature human IVD samples, using DMMB and fixed charge density measurements, and is also noted as an intranuclear cleft with reduced T2 values on MRI (Antoniou et al., 1996; Haughton, 2006; Iatridis et al., 2007).

The mass-spectrometry-based method used is reported as being applied to investigate GAGs in human urine samples and validated using comparisons with DMMB (Schmidt et al., 2016; Sun et al., 2015; Yang et al., 2017). Total GAG as measured using LC-MS is known to correlate with that quantified using DMMB, but the relationship is 
nonlinear (Schmidt et al., 2016). GAG concentrations reported with the DMMB method are greater than for the mass spectrometry method used in the current study, since the DMMB assay measures total GAG and can also detect other polyanions, such as DNA or mucins, resulting in greater background. The mass spectrometry method, used in the current study, relied on multiple reaction monitoring (MRM) mass spectrometry, which was selective and ultra-sensitive. This method was based on disaccharide analysis and used chondroitinase $\mathrm{ABC}$ and heparinases so it could only detect CS/DS, HS, and HA building blocks. Not all disaccharides were fully recovered from purification steps and $\mathrm{KS}$ was also not detected which also contributed to lower concentrations than expected from DMMB. There were sufficient similarities between our data and the literature to validate this mass spectrometry approach, for characterising GAG content in human IVDs, with our suggestion that total DMMB is a simpler method when only total GAG are needed. LC-MS is a more involved method offering specificity of information to characterise GAG types and disaccharides. Furthermore, caution must be exercised when comparing total GAG amounts across methods.

LC-MS was also capable of assessing multiple specific CS and HS disaccharides across different IVD regions and between specimens. Of the $17 \mathrm{CS}$ disaccharides measured, C4S and C6S were found in greatest abundance. C4S and C6S are implicated in inhibiting neurite growth through their presence in NP notochordal cell conditioned media (Purmessur et al., 2015). Furthermore, increases in the C6S:C4S ratio measured in other tissues such as articular cartilage and synovial fluid is considered a strong indicator of joint ageing (Hickery et al., 2003; Nakayama et al., 2002). While the NP generally exhibited similar amounts of each disaccharide, the AF exhibited more C6S than C4S. Interestingly, the young IVD had more C4S than C6S in both NP and AF regions while mature IVDs had more C6S than C4S. Similarly, the C6S:C4S ratio in both NP and AF of the mature human IVDs were increased compared to that of the young. These data suggested a potential shift in CS sulphation with age favouring that of C6S. Notably, a similar inversion of sulphation pattern is observed in another study using bovine IVDs, with higher levels of C4S in immature six-monthold tissue and higher levels of C6S in mature two year-old tissue (Collin et al., 2017). Ovine IVDs also exhibit distinct CS composition in AF and NP with immaturity and maturity (Collin et al. 2016). The distinction of young vs. old GAG disaccharides in the current study was further identified with PCA analysis as the samples with greatest separation. Collectively with the literature, these data suggested the presence of a unique CS sulphation pattern in both NP and AF regions and possibly with age. Thus the use of our GAG analytic method showed potential for identifying changes in CS sulphation unique to ageing, disease and degeneration in the human IVD.

In addition to CS disaccharides, LC-MS also enabled the measurement of HS that, to our knowledge, was the first direct measurement of HS disaccharides in human IVDs. Studies indicate many biological roles for HS, such as that seen in cancer biology (Sasisekharan et al., 2002). Specifically, HS disaccharides in urine are shown to be predictive of acute kidney injury and in-hospital mortality in patients with septic shock or acute respiratory distress syndrome (Schmidt et al., 2016). While HS disaccharides are not generally reported in IVDs, recent studies suggest a role for certain cell surface HS proteoglycans (i.e. syndecan-4 and glypican-6) in AF development, growth and ageing (Beckett et al., 2015; Binch et al., 2016). Our analyses of two predominantly expressed disaccharides in the IVD included that of HOS and H2S. The NP region had similar patterns of HOS and H2S, with the young IVD having greater amounts. In contrast, the AF region of mature IVDs had similar amounts of HOS and H2S while the young IVD had less H2S than HOS. Although these observations were made from a small sample set, the technique enabled distinguishing a strong difference in HS disaccharide patterns between the young and mature specimens, suggesting a possible role in IVD ageing. This potential role could be validated in future studies, using larger sample sizes with wider ranges of age and disease state.

The novel analyses, presented here, generated a large data set quantifying 17 disaccharides on 156 tissue samples providing extensive measurements on IVD GAG types and disaccharides. However, some limitations are important to highlight. These measurements did not determine core proteins, which would require proteomic measurements. This is important, since similar GAG compositions could attach to different proteoglycans to yield distinct functional properties. These analyses did not provide information on disaccharide sequence, quantify KS, or allow distinction between CS and DS, which would all have required additional methodological development. Our study compared mature IVD samples with a single young IVD as a demonstration. This dataset provided estimated differences and variances to perform a power analysis, but did not have sufficient numbers of individual human subjects to statistically test for effects of ageing or degeneration. The results of the current study, along with that available from the literature, point to a potential change in GAG disaccharides with IVD ageing in multiple species; however, it is not clear if there are distinct functional roles for individual GAG disaccharides. GAG disaccharides, core proteins and their sequences may all contribute to functional IVD properties and future studies on their changes with ageing and disease are warranted. 


\section{Conclusion}

This study demonstrated the use of a mass spectrometry technique for the quantitative analysis of GAG disaccharides in human IVD samples. We concluded that the high precision and specificity of LC-MS with MRM was capable of quantifying distribution of CS, HA, HS and their disaccharides across various regions of the IVD. The application of PCA further identified regional patterns that were difficult to assess in this large dataset with traditional statistical analyses. Future studies are required to answer how GAG types and their sulphations change with human IVD ageing and degeneration. It is hoped that improved understanding of the roles of GAG types and sulphation patterns may be useful to distinguish ageing from degeneration processes and perhaps inform novel diagnostic techniques or therapies for painful IVD degeneration.

\section{Acknowledgements}

This work was supported by NIAMS/NIH grant R01AR064157. We gratefully acknowledge the technical assistance of Dr. Paolo Palacio-Mancheno, Melissa Stanley, and Noelle Aly. We would also like to acknowledge Dr. Mary Fowkes and the Mount Sinai Hospital Autopsy Services for contribution of all human specimens.

\section{References}

Acaroglu ER, Iatridis JC, Setton LA, Foster RJ, Mow VC, Weidenbaum M (1995) Degeneration and aging affect the tensile behavior of human lumbar anulus fibrosus. Spine 20: 2690-2701.

Adams MA, Freeman BJ, Morrison HP, Nelson IW, Dolan P (2000) Mechanical initiation of intervertebral disc degeneration. Spine 25: 1625-1636.

Adams MA, Roughley PJ (2006) What is intervertebral disc degeneration, and what causes it? Spine 31: 2151-2161.

Antoniou J, Steffen T, Nelson F, Winterbottom N, Hollander AP, Poole RA, Aebi M, Alini M (1996) The human lumbar intervertebral disc: evidence for changes in the biosynthesis and denaturation of the extracellular matrix with growth, maturation, ageing, and degeneration. J Clin Invest 98: 996-1003.

Barthelemy VMP, van Rijsbergen MM, Wilson W, Huyghe JM, van Rietbergen B, Ito K (2016) A computational spinal motion segment model incorporating a matrix composition-based model of the intervertebral disc. J Mech Behav Biomed Mater 54: 194-204.

Beckett MC, Ralphs JR, Caterson B, Hayes AJ (2015) The transmembrane heparan sulphate proteoglycan syndecan- 4 is involved in establishment of the lamellar structure of the annulus fibrosus of the intervertebral disc. Eur Cell Mater 30: 69-88.
Binch ALA, Shapiro IM, Risbud MV (2016) Syndecan-4 in intervertebral disc and cartilage: Saint or synner? Matrix Biol 52-54: 355-362.

Collin EC, Carroll O, Kilcoyne M, Peroglio M, See E, Hendig D, Alini M, Grad S, Pandit AS (2017) Ageing affects chondroitin sulfates and their synthesis enzymes in the intervertebral disc. Signal Transduct Target Ther. 2: 17049.

Collin EC, Kilcoyne M, White SJ, Grad S, Alini M, Joshi L, Pandit AS (2016) Unique glycosignature for intervertebral disc and articular cartilage cells and tissues in immaturity and maturity. Sci Rep 6: 23062.

Cornejo MC, Cho SK, Giannarelli C, Iatridis JC, Purmessur D (2015) Soluble factors from the notochordal-rich intervertebral disc inhibit endothelial cell invasion and vessel formation in the presence and absence of pro-inflammatory cytokines. Osteoarthritis Cartilage 23: 487-496.

Dieleman JL, Baral R, Birger M, Bui AL, Bulchis A, Chapin A, Hamavid H, Horst C, Johnson EK, Joseph J, Lavado R, Lomsadze L, Reynolds A, Squires E, Campbell M, DeCenso B, Dicker D, Flaxman AD, Gabert R, Highfill T, Murray CJL (2016) US spending on personal health care and public health, 1996-2013. JAMA 316: 2627-2646.

Freemont AJ, Peacock TE, Goupille P, Hoyland JA, O'Brien J, Jayson MI (1997) Nerve ingrowth into diseased intervertebral disc in chronic back pain. Lancet 350: 178-181.

Freemont AJ, Watkins A, Le Maitre C, Baird P, Jeziorska M, Knight MTN, Ross ERS, O’Brien JP, Hoyland JA (2002) Nerve growth factor expression and innervation of the painful intervertebral disc. J Pathol 197: 286-292.

Gao X, Zhu Q, Gu W (2016) Prediction of glycosaminoglycan synthesis in intervertebral disc under mechanical loading. J Biomech 49: 2655-2661.

GBD 2015 Disease and Injury Incidence and Prevalence Collaborators (2016) Global, regional, and national incidence, prevalence, and years lived with disability for 310 diseases and injuries, 1990-2015: a systematic analysis for the global burden of disease study 2015. Lancet 388: 1545-1602.

Gruber HE, Hoelscher GL, Ingram JA, Bethea S, Zinchenko N, Hanley EN (2011) Variations in aggrecan localization and gene expression patterns characterize increasing stages of human intervertebral disk degeneration. Exp Mol Pathol 91: 534-539.

Gunnar Anderson SIW-C (2014) United States bone and joint initiative: the burden of musculoskeletal diseases in the United States (BMUS) Third Edition. Rosemont, IL.

Haughton V (2006) Imaging intervertebral disc degeneration. J Bone Joint Surg Am 88 Suppl2: 15-20.

Hayes AJ, Hughes CE, Ralphs JR, Caterson B (2011) Chondroitin sulphate sulphation motif expression in the ontogeny of the intervertebral disc. Eur Cell Mater 21: 1-14.

Hickery MS, Bayliss MT, Dudhia J, Lewthwaite JC, Edwards JCW, Pitsillides AA (2003) Age-related changes in the response of human articular cartilage 
to IL-1alpha and transforming growth factor-beta (TGF-beta): chondrocytes exhibit a diminished sensitivity to TGF-beta. J Biol Chem 278: 53063-53071.

Hoy D, Brooks P, Blyth F, Buchbinder R (2010) The epidemiology of low back pain. Best Pract Res Clin Rheumatol 24: 769-781.

Iatridis JC, Laible JP, Krag MH (2003) Influence of fixed charge density magnitude and distribution on the intervertebral disc: applications of a poroelastic and chemical electric (PEACE) model. J Biomech Eng 125: $12-24$.

Iatridis JC, MacLean JJ, O'Brien M, Stokes IAF (2007) Measurements of proteoglycan and water content distribution in human lumbar intervertebral discs. Spine 32: 1493-1497.

Iatridis JC, Michalek AJ, Purmessur D, Korecki CL (2009) Localized intervertebral disc injury leads to organ level changes in structure, cellularity, and biosynthesis. Cell Mol Bioeng 2: 437-447.

Iatridis JC, Nicoll SB, Michalek AJ, Walter BA, Gupta MS (2013) Role of biomechanics in intervertebral disc degeneration and regenerative therapies: what needs repairing in the disc and what are promising biomaterials for its repair? Spine J 13: 243-262.

Institute of Medicine (US) Committee on Advancing Pain Research, Care, and Education (2011) Relieving pain in America: a blueprint for transforming prevention, care, education, and research. Washington (DC): National Academies Press (US). The National Academies Collection: Reports funded by National Institutes of Health. doi:10.17226/13172.

Ito K, Creemers L (2013) Mechanisms of intervertebral disk degeneration/injury and pain: a review. Global Spine J 3: 145-152.

Jackson AR, Huang C-YC, Brown MD, Gu WY (2011) 3D finite element analysis of nutrient distributions and cell viability in the intervertebral disc: effects of deformation and degeneration. J Biomech Eng 133: 091006.

Johnson WEB, Caterson B, Eisenstein SM, Hynds DL, Snow DM, Roberts S (2002) Human intervertebral disc aggrecan inhibits nerve growth in vitro. Arthritis Rheum 46: 2658-2664.

Johnson WEB, Caterson B, Eisenstein SM, Roberts $S$ (2005) Human intervertebral disc aggrecan inhibits endothelial cell adhesion and cell migration in vitro. Spine 30: 1139-1147.

Johnson ZI, Schoepflin ZR, Choi H, Shapiro IM, Risbud MV (2015) Disc in flames: Roles of TNF- $\alpha$ and IL-1 $\beta$ in intervertebral disc degeneration. Eur Cell Mater 30: 104-116.

Kadow T, Sowa G, Vo N, Kang JD (2015) Molecular basis of intervertebral disc degeneration and herniations: what are the important translational questions? Clin Orthop Relat Res 473: 1903-1912.

Kiani C, Chen L, Wu YJ, Yee AJ, Yang BB (2002) Structure and function of aggrecan. Cell Res 12: 19-32.

Liu X, St Ange K, Wang X, Lin L, Zhang F, Chi L, Linhardt RJ (2017) Parent heparin and daughter LMW heparin correlation analysis using LC-MS and NMR. Anal Chim Acta 961: 91-99.

Melrose J, Ghosh P, Taylor TK(2001) A comparative analysis of the differential spatial and temporal distributions of the large (aggrecan, versican) and small (decorin, biglycan, fibromodulin) proteoglycans of the intervertebral disc. J Anat 198: 3-15.

Mok FPS, Samartzis D, Karppinen J, Fong DYT, Luk KDK, Cheung KMC (2016) Modic changes of the lumbar spine: prevalence, risk factors, and association with disc degeneration and low back pain in a largescale population-based cohort. Spine J 16: 32-41.

Molinos M, Almeida CR, Caldeira J, Cunha C, Gonçalves RM, Barbosa MA (2015) Inflammation in intervertebral disc degeneration and regeneration. J R Soc Interface 12: 20150429.

Nakayama Y, Narita T, Mori A, Uesaka S, Miyazaki K, Ito H (2002) The effects of age and sex on chondroitin sulfates in normal synovial fluid. Arthritis Rheum 46: 2105-2108.

Purmessur D, Cornejo MC, Cho SK, Hecht AC, Iatridis JC (2013) Notochordal cell-derived therapeutic strategies for discogenic back pain. Global Spine J 3: 201-218.

Purmessur D, Cornejo MC, Cho SK, Roughley PJ, Linhardt RJ, Hecht AC, Iatridis JC (2015) Intact glycosaminoglycans from intervertebral disc-derived notochordal cell-conditioned media inhibit neurite growth while maintaining neuronal cell viability. Spine J 15: 1060-1069.

Rogers CJ, Clark PM, Tully SE, Abrol R, Garcia KC, Goddard WA, Hsieh-Wilson LC (2011) Elucidating glycosaminoglycan-protein-protein interactions using carbohydrate microarray and computational approaches. Proc Natl Acad Sci U S A 108: 9747-9752.

Roughley PJ (2004) Biology of intervertebral disc aging and degeneration: involvement of the extracellular matrix. Spine 29: 2691-2699.

Roughley PJ (2006) The structure and function of cartilage proteoglycans. Eur Cell Mater 12: 92-101.

Samartzis D, Karppinen J, Cheung JPY, Lotz J (2013) Disk degeneration and low back pain: are they fat-related conditions? Global Spine J 3: 133-144.

Sasisekharan R, Shriver Z, Venkataraman G, Narayanasami U (2002) Roles of heparan-sulphate glycosaminoglycans in cancer. Nat Rev Cancer 2: 521-528.

Schmidt EP, Overdier KH, Sun X, Lin L, Liu X, Yang Y, Ammons LA, Hiller TD, Suflita MA, Yu Y, Chen Y, Zhang F, Cothren Burlew C, Edelstein CL, Douglas IS, Linhardt RJ (2016) Urinary glycosaminoglycans predict outcomes in septic shock and acute respiratory distress syndrome. Am J Respir Crit Care Med 194: 439-449.

Sivan SS, Wachtel E, Roughley P (2014) Structure, function, aging and turnover of aggrecan in the intervertebral disc. Biochim Biophys Acta 1840: 31813189.

Stokes IAF, Iatridis JC (2004) Mechanical conditions that accelerate intervertebral disc 
degeneration: overload versus immobilization. Spine 29: 2724-2732.

Su H, Blain F, Musil RA, Zimmermann JJ, Gu K, Bennett DC (1996) Isolation and expression in Escherichia coli of hepB and hepC, genes coding for the glycosaminoglycan-degrading enzymes heparinase II and heparinase III, respectively, from Flavobacterium heparinum. Appl Environ Microbiol 62: 2723-2734.

Sun X, Li L, Overdier KH, Ammons LA, Douglas IS, Burlew CC, Zhang F, Schmidt EP, Chi L, Linhardt RJ (2015) Analysis of total human urinary glycosaminoglycan disaccharides by liquid chromatography-tandem mass spectrometry. Anal Chem 87: 6220-6227.

Thompson JP, Pearce RH, Schechter MT, Adams ME, Tsang IK, Bishop PB (1990) Preliminary evaluation of a scheme for grading the gross morphology of the human intervertebral disc. Spine 15: 411-415.

Uesaka S, Nakayama Y, Yoshihara K, Ito H (2002) Significance of chondroitin sulfate isomers in the synovial fluid of osteoarthritis patients. J Orthop Sci 7: 232-237.

Urban JP, McMullin JF (1988) Swelling pressure of the lumbar intervertebral discs: influence of age, spinal level, composition, and degeneration. Spine 13: $179-187$.

Vo NV, Hartman RA, Patil PR, Risbud MV, Kletsas D, Iatridis JC, Hoyland JA, Le Maitre CL, Sowa GA, Kang JD (2016) Molecular mechanisms of biological aging in intervertebral discs. J Orthop Res 34: 12891306.

Yang Y, Haeger SM, Suflita MA, Zhang F, Dailey KL, Colbert JF, Ford JA, Picon MA, Stearman RS, Lin L, Liu X, Han X, Linhardt RJ, Schmidt EP. (2017) Fibroblast growth factor signaling mediates pulmonary endothelial glycocalyx reconstitution. Am J Respir Cell Mol Biol 56: 727-737.

Yao H, Gu WY (2006) Physical signals and solute transport in human intervertebral disc during compressive stress relaxation: 3D finite element analysis. Biorheology 43: 323-335.

Zhao J, Liu W, Chen D, Zhou C, Song Y, Zhang Y, Ni Y, Li Q (2015) Microbiological and physicochemical analysis of pumpkin juice fermentation by the basidiomycetous fungus Ganoderma lucidum. J Food Sci 80: C241-251.

Zhao X, Yang B, Datta P, Gasmili L, Zhang F, Linhardt RJ (2012) Cell-based microscale isolation of glycoaminoglycans for glycomics study. J Carbohydr Chem 31: 420-435.

\section{Discussion with reviewers}

Abhay Pandit: Are there any examples of individual disaccharides of GAGs having a functional role or are all GAG functions related to disaccharide sequences within the GAG?
Authors: Multiple studies suggest individual disaccharides play distinct functional roles. For example, chondroitin 4-sulphate $(\mathrm{C} 4 \mathrm{~S})$ reduces proinflammatory markers TNF $\alpha$, IL-1 $\beta$ and nitric oxide in LPS-treated mouse chondrocytes in vitro (Campo et al., 2009). Chondroitin 6-sulphate (C6S) disaccharide inhibits neurite growth in embryonic rat neurons (Butterfield et al., 2010). Similarly in the IVD, C6S is abundant in the young notochordal rich-NP tissue and likely contributes to inhibiting neurite growth in rat dorsal root ganglia (known to be involved in innervating IVDs in painful conditions) (Purmessur et al., 2015). CS type E from squid cartilage is shown to exhibit potent antiviral activities, specifically against the herpes simplex virus (Bergefall et al., 2005). While the exact mechanism of action of these GAG disaccharides is still under investigation, the effects were disaccharide specific and some hypothesise that it is due to the positioning of the sulphate groups. For example, sulphation in carbon position 4 of C4S is opposite a carboxylic group and in the midline of the polymer chain, allowing stronger interactions with structures like nitric oxide, contributing to the anti-inflammatory capabilities of C4S. On the other hand, the sulphation at carbon position 6 of C6S occurs more peripherally, thus having a lower affinity for structures like nitric oxide (Campo et al., 2009). GAG sulphation patterns also change in tissue development and maturation, suggesting a potential functional role. For example, the chondroitin 4- sulphation /6- sulphation ratio in embryonic chick brain progressively decreases with development (Kitagawa et al., 1997). The chondroitin 4- sulphation /6- sulphation ratio also changes in human synovial fluid and is used as an indicator of joint ageing and osteoarthritis (Nakayama et al. 2002). In the IVD, sulphation patterns are altered with ageing in bovine and ovine IVDs (Collin et al., 2016; Collin et al., 2017). Collectively these data suggest that individual disaccharides of GAGs and their profiles play functional roles in the IVD and other tissues.

Marianna Peroglio: What differences can one expect with different degrees of degeneration?

Authors: Ageing and degeneration results in GAG loss in the NP of IVDs, but GAG loss with degeneration is described as more rapid and/or of larger magnitude than with ageing (e.g. Sivan et al., 2014; Antoniou et al.,1996). However, very few studies investigated the effects of ageing and degeneration on GAG sulphation patterns. Our results suggested an increase in C6S with ageing in human IVDs, and changes in CS sulphation patterns are also observed with maturing and ageing ovine (Collin et al., 2016) and bovine IVDs (Collin et al., 2017). Our results also exhibited potential age-related shifts in HS sulphation patterns, which are not frequently studied in IVDs. We highlight that ageing and degeneration are distinct processes and hope this method may provide a tool that helps distinguish ageing from degeneration in future studies. 
Marianna Peroglio: The limits of this methodology also lies in assessment of the glycan structures? How does one determine these?

Authors: Our LC-MS method gives important structural information on GAGs, but relies on the removal of GAG chains from core proteins and then the use of chondroitinase ABC. For example, to decompose the CS chains to identify individual CS disaccharides. This precludes us from identifying site-specific glycan structures, their attachment sites as well as analysis of the core proteins themselves. Methodologies to characterise the glycan structures, along with disaccharide compositions, are only starting to be developed. Glycoproteomic methods such as that developed by Nilsson et al. are one recent approach that assesses site-specific CS proteoglycans and was successfully tested on human urine samples. (Nilsson et al., 2017). We previously used 1H-NMR spectroscopy to characterise heparin/HS structure from zebrafish (Zhang et al. 2009). Development of more advanced glycoproteomics methods to advance these techniques would improve knowledge of glycobiology and enable assessments of tissue specific GAGs in diseased states.

Peter Roughley: Do you think the approach used in this study could also be applied to other types of tissues or is it specific to intervertebral disc?

Authors: This approach can be applied to other types of tissues and has been used to successful analyse GAG disaccharides in human urine samples. Using the LC-MS with MRM approach, multiple studies investigating GAG disaccharides in human urine were able to distinguish minute changes in GAG levels from diseased samples such as from patients suffering from mucopolysaccharidoses, acute kidney injury and acute respiratory distress syndrome (Sun et al. 2015; Schmidt et al. 2016). Our study was one of the first to validate this technique on human IVD. Given the success of this approach on both body fluid and tissue samples, it seems promising to study other GAG rich tissues such as cartilage and synovial fluid, both of which have shown strong changes in GAG content with development, age and disease.

Peter Roughley: How are your findings impacting the design of repair strategies for IVD?
Authors: Improving knowledge of glycobiology in the IVD with ageing and degeneration is a fundamental endeavour without a direct translational pathway. GAG compositional changes, including measurements of disaccharides, are likely to have mechanical and biological roles that may inform the development of more specific tissue engineering constructs or therapeutic targets for IVD repair.

\section{Additional references}

Bergefall K, Trybala E. Johansson M, Uyama T, Naito S, Yamada S, Kitagawa H, Sugahara K, Bergstrom T (2005) Chondroitin sulfate characterized by the E-disaccharide unit is a potent inhibitor of herpes simplex virus infectivity and provides the virus binding sites on gro2C cells. J Biol Chem 280: 32193-9.

Butterfield KC, Conovaloff A, Caplan M, Panitch A (2010) Chondroitin sulfate-binding peptides block chondroitin 6-sulfate inhibition of cortical neurite growth. Neurosci Lett 478: 82-7.

Campo GM, Avenoso A, Campo S, D'Ascola A, Traina P, Sama D, Calatroni A (2009) Glycosaminoglycans modulate inflammation and apoptosis in LPS-treated chondrocytes. J Cell Biochem 106: 83-92.

Kitagawa H, Tsutsumi K, Tone Y, Sugahara K (1997) Developmental regulation of sulfation profile of chondroitin sulfate chains in the chicken embryo brain. J Biol Chem 272: 31377-81.

Nilsson J, Noborn F, Toledo AG, Nasir W, Sihlbom C, Larson G (2017) Characterization of glycan structures of chondroitin sulfate-glycopeptides facilitated by sodium ion-pairing and positive mode LC-MS/MS. J Am Soc Mass Spectrom 28: 229-241.

Zhang F, Zhang Z, Thistle R, McKeen L, Hosopama S, Toida T, Linhardt RJ, Page-McCaw P (2009) Strucutral characterization of glycosaminglycans from zebrafish in different ages. Glycoconj J 26: 211218.

Editor's note: The Scientific Editor in charge of this paper was Mauro Alini. 